\title{
Low-Cost-Sensoren für die VOC Detektion
}

\author{
${ }^{1}$ Rosemarie Dittrich, ${ }^{2}$ Andreas Ostman, ${ }^{1}$ Yvonne Joseph, \\ ${ }^{1}$ Institut für Elektronik- und Sensormaterialien, TU Bergakademie Freiberg, \\ ${ }^{2}$ Fraunhofer IZM, Berlin, Deutschland \\ Kontakt: rosemarie.dittrich@esm.tu-freiberg.de, +493731392644
}

\section{Zusammenfassung}

Die Anwendung chemischer Sensoren in den Lebenswissenschaften und im medizinischen Bereich zeichnet sich durch den schnellen Informationsgewinn, die nicht-invasive Anwendung und den Einmalgebrauch aus. Die Sensoren müssen daher kostengünstig hergestellt werden können und dabei hohe Empfindlichkeiten sowie anwendungsbezogene Selektivitäten aufweisen. Insbesondere die Aufbau- und Verbindungstechnik (AVT) ist bisher sehr teuer. Zur Realisierung kostengünstiger Sensoren wurden die einkristallinen oxidierten Si-Wafersubstrate durch kommerzielle polymere Leiterplattenmaterialien ersetzt und als Elektrodenmaterial Silber statt Gold eingesetzt. Auf diese sind selbstorganisierende Nanokomposite auf Basis von Gold-Nanopartikeln und verschiedenen organischen Molekülen in Form von ultradünnen Schichten aufgebracht worden [1]. Die abgeschiedenen Schichten wurden licht- und rasterelektronenmikroskopisch, mittels Photoelektronenspektroskopie (XPS) und elektrischer Messungen zur Ermittlung der sensorischen Eigenschaften untersucht.

Schlagwörter: Low cost Sensoren, Leiterplattensubstrate, Au-Nanopartikel, Selbstassemblierung

\section{$1 \quad$ Prozessierung}

\subsection{Substrate}

Als Substrate für eine low-cost-Technologie wurden Polyurethan (PU), Polyimid (PI) und EpoxyLeiterplattenmaterial (ELP) untersucht. Für die Prozessierung des Sensos ist die chemische und thermische Beständigkeit des Materials von großer Bedeutung. Chemisch beständig sollten die Materialien gegenüber den bei der Sensorprozessierung eingesetzten Lösungsmitteln sein, die je nach Schichtmaterial Ethanol, Aceton und Toluol sein können. Die Substrate wurden bis zu 24h in den Lösungsmitteln gelagert und anschließend unter einem Argongasstrom getrocknet und optisch geprüft. Dabei zeigte sich, dass sowohl PI als auch ELP geeignet sind. Da sich die Ergebnisse von PI und ELP nicht signifikant unterschieden (XPS und sensorische Messungen), werden im Paper nur die Ergebnisse von Epoxy-Leiterplattenmaterial dargestellt.

\subsection{Schichtmaterialien}

Als Schichtmaterialien für die sensorischen Messungen wurden Au- Nanopartikeln (AuNP) mit verschiedenen organischen Linkern auf den mit Elektroden beschichteten Leiterplattensubstraten abgeschieden. Die $\mathrm{Au}$ - Nanopartikel besitzen einen mittleren Durchmesser von $4 \mathrm{~nm}$ und liegen in Toluol dispergiert vor. Für die Präsentation wurde $5 \mathrm{mM}$ Nonandithiol (NDT) (HS- $\left.\left(\mathrm{CH}_{2}\right)_{9}-\mathrm{SH}\right)$ als Linkermaterial ausgewählt.

\subsection{Substratherstellung}

Die Substrate mit den Elektrodenstrukuren wurden in einer Nutzengröße von 310x228 mm² hergestellt. Hierfür wurde eine $2 \mu \mathrm{m} \mathrm{Cu}$-Folie mit einem $250 \mu \mathrm{m}$ starken Epoxyd-Material („Prepreg“) mittels VakuumLamination verbunden. Zur Verbesserung der Handhabung ist die sehr dünne $\mathrm{Cu}$-Schict mit einer dickeren $\mathrm{Cu}$-Trägerfolie verbunden, die nach dem Laminierprozeß abgezogen werden kann. Auf die $2 \mu \mathrm{m} \mathrm{Cu}$-Schicht wurde eine fotoempfindliche Resistschicht mittels eines Rollenlaminators aufgetragen. Die Belichtung der Schicht mit UV-Licht der Wellenlänge $355 \mathrm{~nm}$ erfolgte maskenlos in einem Laserdirektbelichter (LDI). Das eingesetzte System Obotech Paragon Ultra 200 erlaubt eine minimale Strukturauflösung von $10 \mu \mathrm{m}$. Nach der Belichtung würde der Resist in einer alkalischen Lösung entwickelt. Anschließend erfolgte der Abtrag des freiliegenden $\mathrm{Cu}$ in einer Durchlaufanlage mit saurer $\ddot{\text { Ätz- }}$ lösung. Danach wurde der Fotoresist in alkalischer Lösung gestrippt. Die metallische $\mathrm{Cu}$-Oberfläche kann leicht oxidieren und ist damit als Elektrodenoberfläche für chemische Sensoren ungeeignet. Daher wurde eine ca. $0,1 \mu \mathrm{m}$ starke Ag-Schicht in einem Tauchprozeß auf dem $\mathrm{Cu}$ abgeschieden.

\subsection{Beschichtung}

Für die Abscheidung der Au-Nanopartikel wurde ein Qsense E1 QCM-D System (Abb.1) benutzt. Durch automatisierte lagenweise Abscheidung mittels Selbstassemblierung im Durchflussprinzip konnten ohne weitere 
Vorbehandlung Au-Nanopartikelschichten auf den Substraten realisiert werden (siehe Schema in Abb.2).

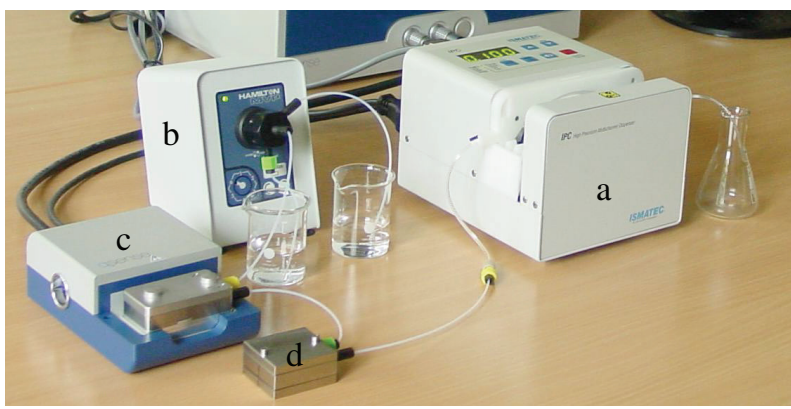

Abb.1: Versuchsaufbau zur Beschichtung mit AuNP a) Peristaltikpumpe, b) Ventil, c) QCM-Zelle, d) Beschichtungszelle

Dabei wurde ein Schichtkomposit aus AuNP und NDT realisiert, indem abwechselnd mit einer Durchflussrate von $100 \mu 1 / m i n$ beschichtet wurde. Zwischen jeder Beschichtung wurde mit Toluol gespült, um unvernetzte Reste der Beschichtungsmaterialien zu entfernen.

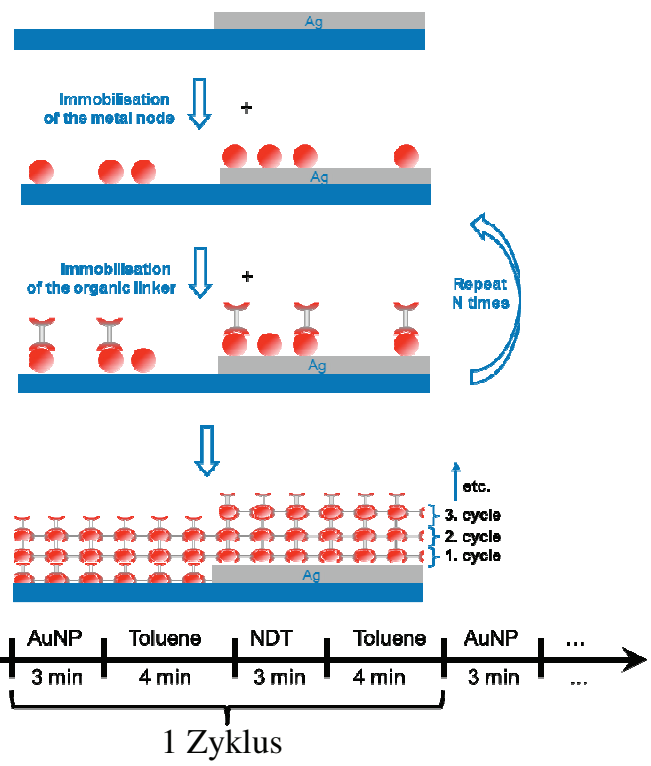

Abb.2: Layer by layer Selbstassemlierung von AuNP mit organischen Linkern

Abb.3 zeigt eine abgeschiedene Schicht von AuNP auf einem Leiterplattensubstrat mit Ag-Kontakten und entsprechender interdigitaler Elektrode unter der Schicht. Der Elektrodenabstand betrug $20 \mu \mathrm{m}$ und die Elektrodenfläche $6 \times 4 \mathrm{~mm}$. Eine weitere Miniaturisierung der Elektrodenfläche wird zur Zeit untersucht.

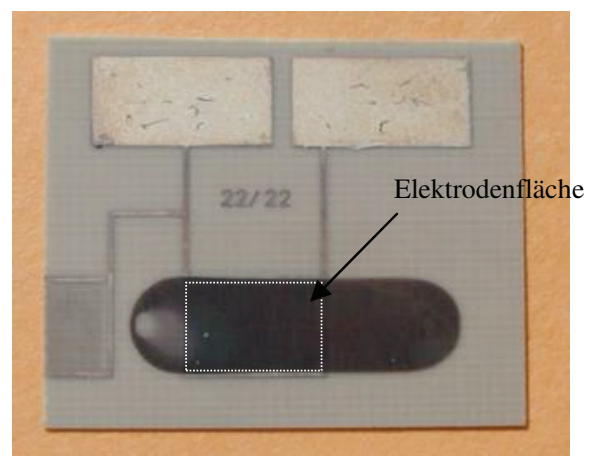

Abb.3: AuNP-Schicht mit Ag-Kontaktierung auf EpoxyLeiterplattensubstrat

\section{Charakterisierung}

2.1 Mikroskopische Untersuchungen

Sowohl die unbeschichteten als auch die beschichteten Substrate wurden licht- als auch elektronenmikroskopisch untersucht. Es mussten mehrere Schichten abgeschieden werden, um eine geschlossene Schichtstruktur zu erzeugen. In unserem Fall wurden jeweils 10 Schichten aufgebracht.

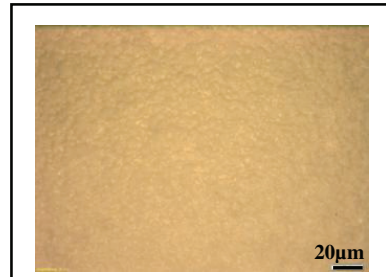

a

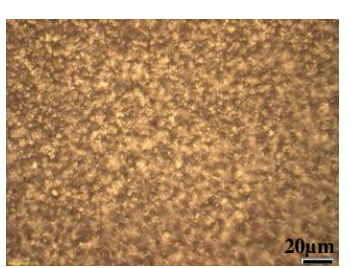

b
Abb.4: Lichtmikroskopische Aufnahmen des unbeschichteten (a) und des mit AuNP-NDTbeschichteten (b) Epoxy-Leiterplattenmaterials

Die Bilder in Abb.3 und Abb.4 zeigen, dass nach der Abscheidung eine homogene feinteilige Schichtoberfläche vorliegt.

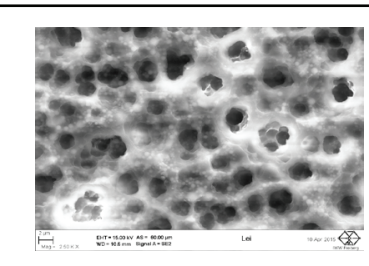

a

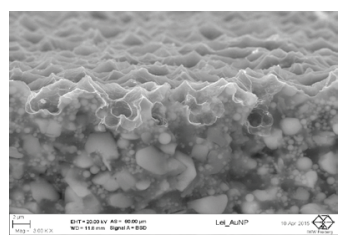

b
Abb.5: REM-Aufnahmen des

(a) unbeschichteten Substrates (Oberfläche) und (b) des mit AuNP-NDT-beschichteten Substrates (Querschnitt) aus Epoxy-Leiterplattenmaterial 
Mittels rasterelektronenmikroskopischer Untersuchungen konnte an einer Bruchfläche die AuNP- Schicht dargestellt werden, die sich dünn auf dem Leiterplattensubstrat gebildet hatte (Abb.5).

\subsection{Photoelektronenspektroskopische Untersuchungen (XPS)}

Mittels XPS wurde die chemische Zusammensetzung der abgeschieden AuNP-NDT-Kopmpositschicht untersucht, wobei die Elemente $\mathrm{Au}, \mathrm{C}, \mathrm{S}$ und $\mathrm{O}$ ausgewertet wurden.

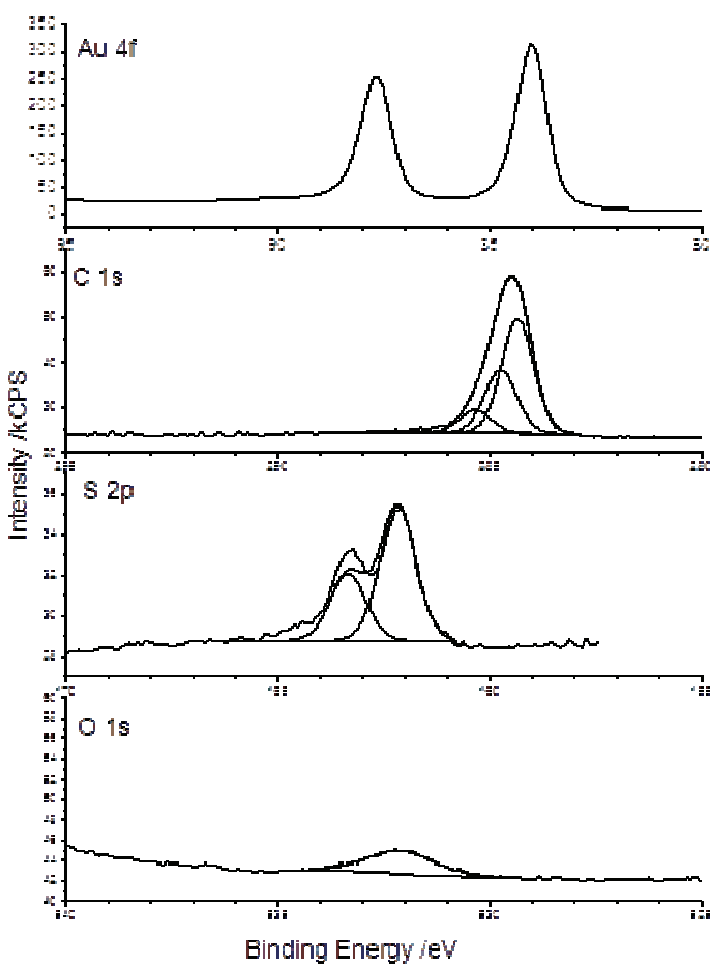

Abb.6: Ergebnisse der XPS-Untersuchungen an AuNPNDT-Schichten auf Epoxy-Leiterplattenmaterial

Ein Vergleich mit konventionellem Substratmaterial (Glas) (Abb.7) zeigte, dass durch das Substrat nur ein geringer Einfluss auf die chemische Zusammensetzung der Schicht registriert werden konnte (AbbX).

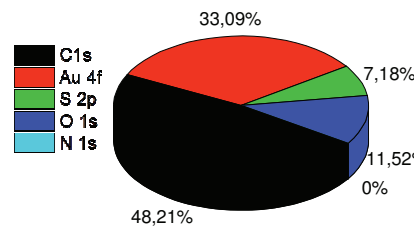

Glas

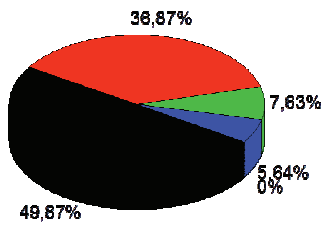

Epoxy-Leiterplatte
Abb.7: XPS-Ergebnisse an AuNP-NDT-Schichten auf verschiedenen Substraten

\subsection{Sensorische Messungen}

Für die sensorische Charakterisierung kamen vier volatile organische Komponenten (VOC) (Wasser, Toluol, 1Propanol und 4-Methyl-2-Pentanon (4M2P)) zum Einsatz, die über Sättiger mit trockener Luft gemischt in verschiedenen Konzentrationen als Gas über das Schichtmaterial geleitet wurden. Alle Proben wurden bei Konzentrationen zwischen 100 und 5000 ppm des Analyten in drei Zyklen von jeweils 120s mit dem Gas beaufschlagt. Zwischen den Zyklen wurde mit trockener, gereinigter Luft ebenfalls 120s gespült.

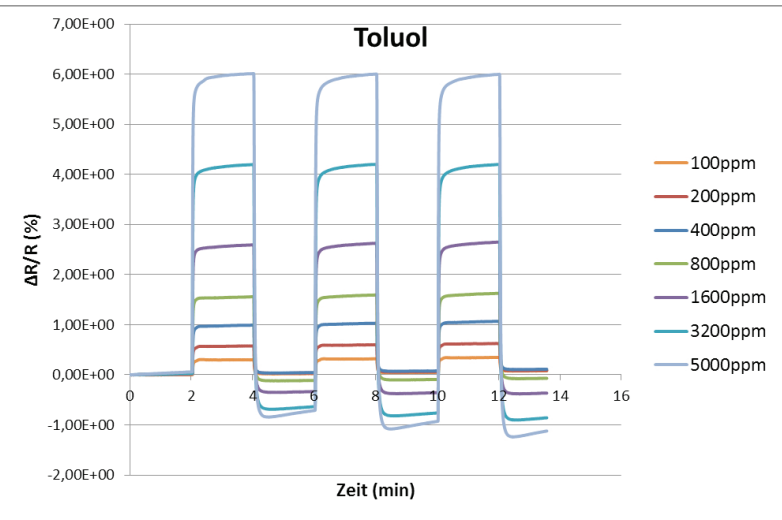

Abb.8: Widerstandänderung der AuNP-NDT-Schicht in Abhängigkeit von der Konzentration an Toluol im Messgas

Wie die Messkurven zeigen, besitzen die Sensoren sehr kurze Ansprechzeiten von wenigen Sekunden (Abb.810). Der Austausch des Analyten gegen trockene Luft erfolgte ebenfalls innerhalb weniger Sekunden. Sowohl mit Toluol als auch mit Wasser traten Driften des Messsignals mit zunehmender Zyklenzahl auf, die möglicherweise darauf zurückzuführen sind, dass Analyt in das Substrat diffundiert, was jedoch noch näher untersucht werden muss.

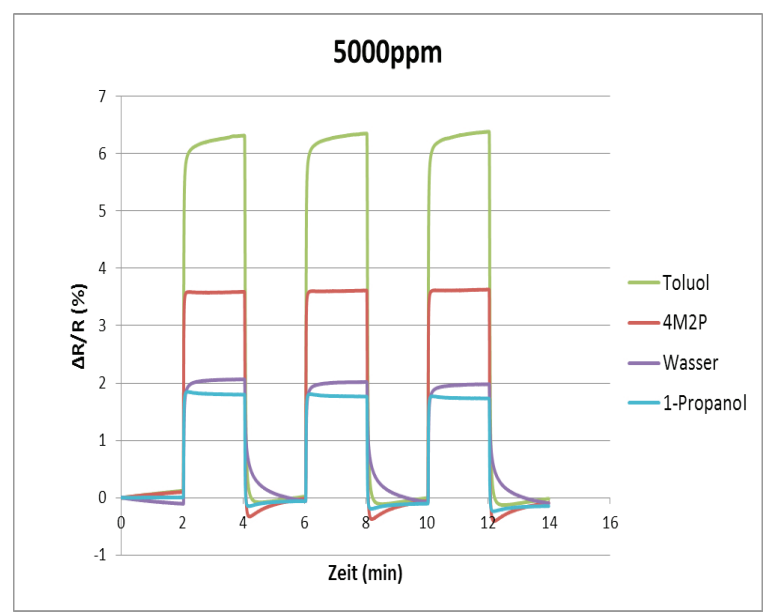

Abb.9: Widerstandsänderung der AuNP-NDT-Schicht in Abhängigkeit von der Art des Analyten bei Konzentrationen von 5000ppm 


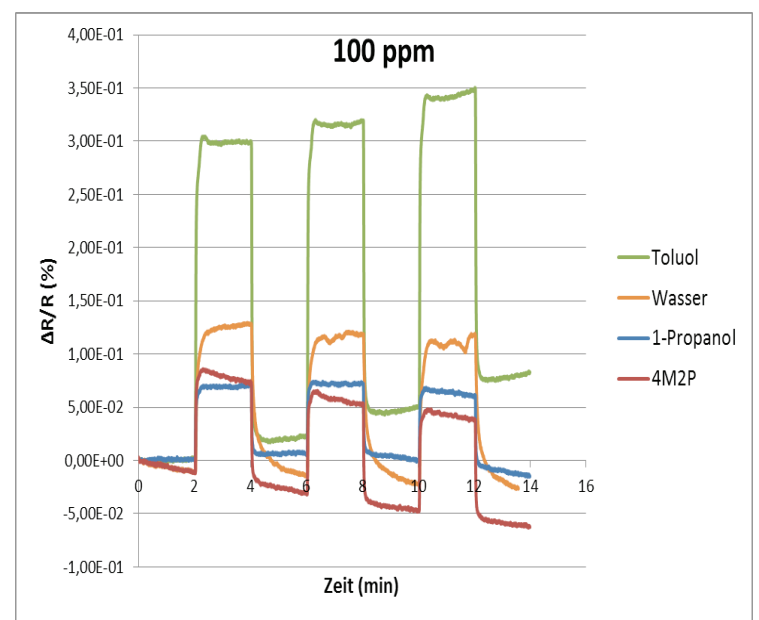

Abb.10: Widerstandsänderung der AuNP-NDT-Schicht für verschiedene Analyte bei einer Konzentration von $100 \mathrm{ppm}$

Alle untersuchten Analyte konnten bei 100 ppm noch detektiert werden. Eine Abschätzung ergibt, dass dieser Schichtaufbau bis zu Analytkonzentrationen von 20 ppm anwendbar sein sollte.

Um den Einfluss der Substratmaterialien auf die sensorischen Eigenschaften weiter $\mathrm{zu}$ untersuchen wurden AuNP-NDT-Schichten sowohl auf Si-Wafern als auch auf Epoxy-Leiterplattenmaterial durch Selbstassemblierung wie beschrieben prozessiert.

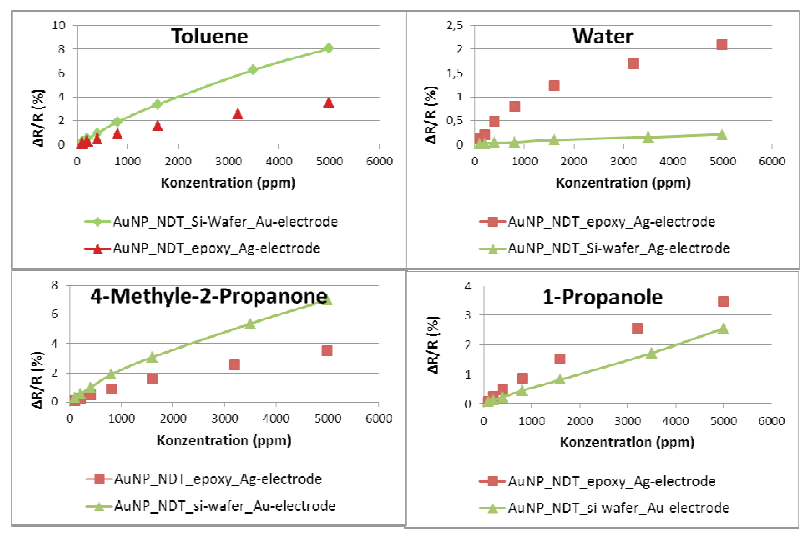

Abb.11: Vergleich der sensorischen Eigenschaften von Au-NP-NDT-Komposite auf unterschiedlichen Substraten

Es zeigte sich (Abb.11), dass tendenziell ähnliche Kurvenverläufe in Abhängigkeit von der Analytart entstanden. Die größten Unterschiede wurden bei den Messungen mit Wasser registriert, was auf die poröse Struktur des Epoxy-Leiterplattenmaterials zurückzuführen sein kann.

\section{Literatur}

[1] Joseph Y, Besnard I, Rosenberger M, Guse B, Nothofer HG, Wessels JM, Wild U, Knop-Gericke A, Su D, Schlögl R, Yasuda A, and Vossmeyer T;Self-Assembled Gold Nanoparticle/Alkanedithiol Films: Preparation, Electron Microscopy, XPS-Analysis, Charge Transport and Vapor-Sensing Properties; J. Phys. Chem. B 107, 7406-7413 (2003). 